\title{
CONCEPÇÕES E EXPERIÊNCIAS EM CUIDADOS PALIATIVOS NO NORTE DE MINAS GERAIS
}

\section{CONCEPTIONS AND EXPERIENCES IN PALLIATIVE CARE: IN THE NORTH OF MINAS GERAIS}

\author{
Sandra Célia Muniz Magalhães \\ Doutora em Geografia
} sandramunizgeo@hotmail.com

Dardielle Cardoso de Oliveira Graduada em Geografia dardielleco@gmail.com

Priscila Bernardina Miranda Souza Mestre em Ciências da Saúde priscilamirandasoares@yahoo.com.br

Flávia de Oliveira Santos Doutora em Geografia flaviasantos1@yahoo.com.br

Isabella Giovanna Sá Cirurgiã-dentista

\section{RESUMO}

O termo paliativo tem origem no latim, pallium, que teoricamente expressa manto e capote. Os cuidados paliativos são os auxílios disponibilizados por um quadro de profissionais multidisciplinar, que atuam na assistência terminal, em que almeja aumentar a qualidade de vida do doente terminal e de seus familiares diante de um adoecimento que ameace a sua vida. Esse auxílio acontece através da prevenção e atenuação do suplício, mediante a constatação precoce, avaliação cuidadosa, tratamento da dor e de outras adversidades físicas, psicossociais e espirituais. Neste contexto, esse trabalho tem o objetivo de discutir algumas concepções acerca dos cuidados paliativos, além de apontar experiências nesse cuidado realizadas pela Associação Presente, na região Norte de Minas Gerais. Para tanto, a metodologia utilizada consistiu em pesquisa bibliográfica e documental, as bases de informação foram LILACS e SciELO, sendo escolhidos 36 artigos, publicados entre 2000 a 2006, e entrevistas com pacientes e equipe multiprofissional da Associação Presente. Foram desenvolvidas definições sobre a terminalidade da vida, a adequação da comunicação frente às barreiras, sendo classificada como essencial a aceitação da morte, como um fenômeno normal e o respeito ao paciente. Considera-se que estas concepções assumem grande importância nos cuidados paliativos, onde na fase final da vida são indispensáveis e se estendem até o estágio de luto, de maneira diferenciada. Assim, a Associação Presente presta serviço fundamental para o acolhimento das pessoas nessa condição.

Palavras chave: Cuidados Paliativos. Doente. Assistência paliativa. Final da Vida.

\footnotetext{
${ }^{2}$ Agradecimentos ao CNPQ.
}

Recebido em: 11/05/2019

Aceito para publicação em: 07/10/2019 


\begin{abstract}
The term palliative comes from the Latin, pallium, which theoretically expresses mantle and cloak. Palliative care is the aid provided by a multidisciplinary professional staff, who work in terminal care, in which it aims to increase the quality of life of the terminally ill patient and his family in the face of an illness that threatens his life. This aid comes through the prevention and mitigation of torture, through early detection, careful evaluation, treatment of pain and other physical, psychosocial and spiritual adversities. In this context, this work has the objective of discussing some conceptions about palliative care, besides pointing out experiences in this care carried out by Associação Presente, in the Northern region of Minas Gerais. For that, the methodology used consisted of bibliographic and documentary research, the information bases were LILACS and SciELO, being chosen 36 articles, published between 2000 to 2006, and interviews with patients and multiprofessional team of the Present Association. Definitions have been developed about the termination of life, the adequacy of the communication in front of the barriers, being classified as essential the acceptance of death, as a normal phenomenon and respect to the patient. It is considered that these conceptions assume great importance in palliative care, where in the final phase of life are indispensable and extend to the stage of mourning, in a differentiated way. Thus, the Gift Association provides a fundamental service for the reception of people in this condition.
\end{abstract}

Key works: Palliative care. Sick. Palliative care. End of Life.

\title{
INTRODUÇÃO
}

Desde a antiguidade, assuntos associados ao processo saúde-doença sempre foi o foco da atenção humana, sendo notório que a exposição a inúmeros aspectos no prelúdio, eram identificados como punição de forças divinas. Com o avanço do conhecimento cientifico, a compreensão da sociedade foi transformada por intermédio das pesquisas que procuravam entender as diversas doenças que se manifestaram em distintas épocas. De acordo com Câmara et al (2012, p. 41):

As doenças crônicas podem se desenvolver a partir de modos de vida, sendo considerados fatores de risco a alimentação inadequada, a falta de atividade física e o tabagismo. O sedentarismo tem sido considerado como o fator de risco mais prevalente na população, independentemente do sexo. Associada a pouca atividade física, a alimentação inadequada vem contribuindo para aumentar o número de obesos,

Deste modo, por meio dos fatores ambientais efetivou-se a vinculação entre o conhecimento geográfico e os estudos da saúde, já que o espaço seu objeto de pesquisa, segundo Jayme (2013,p.20):

[...] têm ganhado ênfase nos estudos da saúde seja como um simples plano geométrico para disposição dos dados epidemiológicos ou como uma aproximação dos fatores sócioespaciais no que concerne a promoção e proliferação das doenças, bem como, na identificação das áreas de risco e consequentemente sua causa e o efeito.

O conhecimento cientifico em especial na medicina atravessou inúmeras modificações no decorrer dos anos. Com o desenvolvimento da prática médica paliativa, aliada a tecnologia e as novas concepções oferece evolução considerável os cuidados paliativos, na atenuação do suplicio das doenças terminais, já que não há possibilidade de cura. A atenuação do suplício, a benevolência pelo enfermo e suas famílias, a monitoração dos sintomas e da dor, a procura pela independência e pela conservação da qualidade de vida, são algumas das convecções dos Cuidados Paliativos, introduzido em diversas áreas do corpo social brasileiro. Nesse interim, os Cuidados Paliativos são tratamentos que aumenta a qualidade de vida de doentes e seus familiares perante doenças que atemoriza a conservação da vida. 
Diante do exposto, o presente trabalho tem por objetivo discutir algumas concepções acerca dos cuidados paliativos, além de expor as ações realizadas pela Associação Presente no Norte de Minas Gerais nessa direção.

\section{CONCEITUAÇÃO E HISTÓRIA DOS CUIDADOS PALIATIVOS}

As profundas transformações do sistema do enfermar e falecer ocasionadas pelas modificações populacionais, da filosofia do conhecimento e estudo das doenças, e com o crescente avanço da qualidade e da expectativa de vida, mas também o aumento das enfermidades crônicas e degenerativas levou ao centro da atenção as indagações éticas a respeito dos cuidados possibilitados para os doentes terminais. Sendo que o paciente terminal é o personagem principal desse processo de falecer, tendo um papel ativo nas ações e decisões do tratamento.

De acordo com a filologia o termo "paliativo" tem origem do latim pallium, que expressa "coberto com capa", "manto". Pallium era o manto utilizado pelos romeiros ou peregrinos para se defender e preservar das adversidades no decorrer das viagens a caminho dos templos religiosos. Em relação, ao cuidado paliativo tem como finalidade resguardar os pacientes do suplicio, preservando sua integridade como ser humano até a terminalidade da vida. Segundo Pessini $(2001 ; 2004)$, o preludio dos Cuidados Paliativos remonta ao século IV. Conforme o autor, o auxílio para com os doentes sem expectativas terapêuticas curativas é um tanto pretérito, existente desde a Antiguidade. Nesse interim, pode- se ressaltar que o que existia em tempos remotos no quesito de atenção e ajuda ao indivíduo na terminalidade da vida se difere basicamente do que compreendemos por Cuidados Paliativos na atualidade.

$\mathrm{Na}$ Antiguidade, o auxílio aos enfermos era praticado por pessoas simples que, por compaixão, propiciavam asilo aos doentes em suas moradias. Segundo Pessini $(2001$; 2004), posteriormente, a atenção e a ajuda aos excluídos do convívio social devido às enfermidades graves e em estágio terminal foram adquiridos pela Igreja, o que estendeu por toda a Idade Média. Apenas muito anos depois, já no século XX, os Cuidados Paliativos se converteram em uma atividade de saúde institucionalizada e se manifestaram como uma nova categoria de auxílio aos pacientes terminais, quando não há mais possibilidades curativas.

Isto aconteceu em Londres, na Inglaterra, com a constituição do primeiro hospice ( lugar onde ocorre a realização da atividade dos Cuidados Paliativos com doentes em fase terminal, busca conservar um ambiente familiar, com sociabilização dos pacientes, horário de visitas, autorização para o comparecimento de pessoas e animais) moderno "o St. Christopher" em 1967, por Cicely Saunders, que tem a formação médica, enfermeira e assistente social. Declarada, por conseguinte, uma pioneira nos Cuidados Paliativos, a mesma empenhou sua vida em benefício do auxílio a humanização para os enfermos oncológicos na terminalidade da vida, com a finalidade de disponibilizar um falecimento apropriado. Conforme Menezes (2004, p. 53), o St. Christopher's hospice "[...] transformou-se em modelo de assistência, ensino e pesquisa no cuidado dos pacientes terminais e de suas famílias".

Anteriormente ao hospice do St. Christopher's foram criados outros, mas sem preocupação com a qualidade de vida e a atenuação do suplicio do doente terminal. Entretanto, com a atividade pioneira de Dame Cicely Saunders e com a criação do St. Christopher's Hospice que deu abertura ao Movimento Hospice Moderno, no qual deu origem aos atuais Cuidados Paliativos. Segundo Pessini e Bertachini (2005, p. 497):

Cicely Saunders começou sua carreira profissional primeiro como enfermeira e assistente social. Depois estudou medicina, para segundo ela mesma "cuidar bem dos pacientes terminais, esquecidos pelos médicos tradicionais". Ela é reconhecida como a fundadora do movimento moderno de hospice. $O$ St.Christopher's Hospice por ela fundado foi o primeiro hospice que, numa visão holística da pessoa humana e cuidados integrados, ligou o alívio da dor e controle de sintomas com o cuidado humanizado, o ensino e a pesquisa clínica. Essa nova filosofia de cuidados direcionada aos pacientes fora de possibilidades terapêuticas influenciou muito os cuidados em saúdeao redor do mundo, bem como gerou novas atitudes em relação à morte, ao morrer e diante da dor da perda de um ente querido, isto é, o período do luto.

A ideologia de cuidados aos enfermos terminais utilizados por Dame Cicely Saunders estimulou o surgimento de outros hospices, que não operavam como lugares de abandonar os enfermos. Os 
hospices modernos foram criados para assegurar qualidade de vida aos doentes terminais. Para realizar esta finalidade, é imprescindível auxílio de um grupo especializado e multidisciplinar. De acordo com Menezes e Heilborn, (2007, p. 571) "A influência do fenômeno Nova Era na proposta dos Cuidados Paliativos é patente, especialmente quanto à visão da vida como um fluxo, no qual está inserido um indivíduo único e singular, cuja interioridade é considerada como locus de sua verdade". Já para Rodriguéz (2002), esta constituição possibilita o aperfeiçoamento de projetos de cuidado integral às necessidades dos pacientes e de seus familiares, em suas perspectivas físicas, emocionais, sociais e espirituais.

Gradativamente, os Cuidados Paliativos foram se incorporando as organizações de saúde e se expandiram por todo o mundo. Uma espécie de divisor de aguas para sua legalização foi a definição realizada pela Organização Mundial de Saúde (OMS), o que aconteceu no ano de 1990, mais de duas décadas depois da formação da ação paliativista moderna. Em 1990, a OMS definiu, pela primeira vez, o cuidado paliativo como, "[...] o cuidado total e ativo de pacientes cuja doença não é mais responsiva ao tratamento curativo. São da maior importância: o controle da dor e outros sintomas, como também os psicológicos, espirituais e sociais". Esse processo propiciava segundo Pessini (2006, p. 63), "[...] um sistema de suporte que ajude os pacientes a viver o mais ativa e criativamente possível até a morte". Em 2002, essa definição foi ratificada e a Organização Mundial de Saúde - OMS (2016), determinou os Cuidados Paliativos como:

um conjunto de medidas que visam à melhoria da qualidade de vida de pacientes e familiares que se deparam com questões relacionadas a uma doença ameaçadora da continuidade existencial, através da prevenção e do alívio do sofrimento possibilitados pela identificação precoce, pela eficiente avaliação e tratamento da dor, bem como pela atenção a outros sintomas físicos, psíquicos e espirituais.

O processo dos cuidados de atenuação deve- se congregar as habilidades de um quadro de funcionário multidisciplinar para auxiliar o doente a adequar-se as alterações de vida determinada pela enfermidade, e favorecer a meditação e a analise essencial para o enfrentamento da doença que ameaça a vida do paciente. Entretanto, para que esta atividade seja efetuada é fundamental possuir uma equipe mínima de profissionais, constituída por: um médico, uma enfermeira, uma psicóloga, uma assistente social e pelo menos um profissional da área da reabilitação. Todos apropriadamente preparados na filosofia e realização da paliação. Segundo Capelas (2009, p.51):

Os cuidados paliativos consideram-se, hoje em dia, um direito humano. Neste ponto de vista a implementação dos mesmos deve obedecer a uma estratégia e não a uma desordenada pulverização de recursos. Qualquer estratégia tem como ponto de partida uma avaliação das necessidades, que quando não se consegue fazer de forma real, se poderá estimar.

No Brasil, em janeiro de 2002 foi publicado pelo Ministério da Sade (MS), a portaria GM/MS n. 19 , que tinha como objetivo desenvolver, no Sistema Único de Saúde (SUS), o Programa Nacional de Assistência à Dor e Cuidados Paliativos, cujas finalidades gerais são: associar atividades governamentais ou não direcionas ao cuidado aos doentes nos cuidados paliativos; estruturar serviços e equipes multiprofissionais para a atenção aos doentes que precisam dos cuidados paliativos para constituir um ambiente organizado; propiciar ações direcionadas a prática assistencial, a instrução continua das equipes de saúde e a instrução social para a atenção nos cuidados paliativos; aprimorar atividades na qualificação e difusão de informações importantes para equipes de assistência a saúde, doente, familiares e sociedade sobre a realidade epidemiológica, estudos, assistências, cuidados paliativos, dentre outros; aprimorar regras de auxílios nacionais, adequadas a atualidade da população brasileira, disponibilizar cuidados apropriados aos doentes sem possibilidade da cura de acordo com as regras internacionais organizadas pelas associações de saúde e sociedade comprometidas com a temática.

Já a portaria GM/MS n 2.439 , de 8 de dezembro de 2005 reproduziu um desenvolvimento na constitucionalização política e social dos Cuidados Paliativos no Brasil. A mesma elaborou a Política Nacional de Atenção Oncológica e incluiu os Cuidados Paliativos como componente indispensável a um auxílio intermitente aos doentes com câncer, em conjunto com a atenção, o estudo da patologia, e o tratamento. Em 12 de dezembro de 2006, mais um avanço foi atingido com a consolidação da 
Câmara Técnica em Controle da Dor e Cuidados Paliativos por intermédio da portaria GM/MS n 3.150 .

Em 1997, foi criada em São Paulo a Associação Brasileira de Cuidados Paliativos (ABCP) com a finalidade de desenvolver e propiciar os Cuidados Paliativos em enfermidades crônico-evolutivas durante a terminalidade, por mediação da constituição de profissionais de saúde, fornecendo auxilio e o desenvolvimento de estudos científicos. Em fevereiro de 2005, uma equipe de especialistas, insatisfeitos com a falta de representatividade dos paliativistas no Brasil, criou a Academia Nacional de Cuidados Paliativos (ANCP), associação profissional que tem como missão promover o reconhecimento da Medicina Paliativa como especialidade pelas entidades médicas competentes.

\section{ASSOCIAÇÃO PRESENTE DE APOIO A PACIENTES COM CÂNCER - PADRE TIÃOZINHO}

A Associação Presente de Apoio a Pacientes com Câncer - Padre Tiãozinho do Norte de Minas Gerais inicia suas atividades em 2004, em que o Padre Sebastião Raimundo de Castro em tratamento contra o câncer e sua oncologista a Doutra Priscila Bernardina Miranda Soares decidiam desenvolver o Projeto Presente, para amparar o paciente carente em tratamento oncológico na Região Norte de Minas Gerais. Atualmente, já realizado, denomina-se Associação Presente. A Essa Associação já auxiliou mais de 3.000 pacientes por meio de doação de medicamentos, perucas, roupas, cestas básicas, apoio psicológico, jurídico e espiritual, e campanhas de conscientização para a prevenção e diagnóstico precoce.

No dia 28 de Junho de 2013 foi inaugurada no bairro Canelas uma sede própria, em Montes Claros/MG, com $791 \mathrm{~m}^{2}$ de área construída, com a ajuda da comunidade através de doações para acolher os pacientes carentes. Possui uma infraestrutura privilegiada com: 32 leitos para pacientes e acompanhantes organizados em oito quartos, que proporcionam maior conforto aos que enfrentam a enfermidade; uma capela; área administrativa; espaço para auxilio psicológico, social, nutricional, jurídico e espiritual para os pacientes e seus familiares; e uma vasta área de contato para a prática artesanal, reflexões e palestras instrutivas.

A Associação Presente tem como missão oferecer auxilio, cuidado, atenção e apoio a pacientes carentes de todas as faixas etárias com câncer e agir na prevenção e diagnóstico precoce da doença. Apoiar cidadãos provenientes de cidades de todo o Norte de Minas, que buscam em Montes Claros/MG o tratamento para o câncer proporcionando ajuda e amparo a pacientes, e seus familiares e acompanhantes, no desenvolvimento de projetos de conscientização pública, por meio da difusão de informação sobre a instrução em saúde e proteção aos direitos dos pacientes oncológicos. E ser modelo de instituição filantrópica e símbolo de solidariedade e humanidade de amparo a pacientes oncológicos na Região Norte de Minas Gerais.

A alguns dos serviços disponibilizados são: apoio e hospitalidade; assistência da equipe multiprofissional, com visitas diárias tanto na Associação Presente como em domicilio; oferecimento de refeições diárias aos pacientes e seus acompanhantes; transporte para hospitais, clinicas e ambulatórios; uso de terapias ocupacionais para o desenvolvimento da saúde; e realização de mutirões e encontro para a prevenção do câncer e identificação precoce da enfermidade, além dos cuidados paliativos a pacientes com a proximidade da finitude da vida.

\section{PROCEDIMENTOS METODOLÓGICOS}

Constituiu-se inicialmente de um estudo bibliográfico em que foram empregados dados e informações online (LILACS e SciELO). Desta maneira, a princípio foi efeituada pesquisa sobre os estudos relativos aos cuidados paliativos, tendo como finalidade identificar as concepções sobre o procedimento de zelar. Na verificação preliminar foram observados títulos e resumos dos artigos científicos para uma apuração geral (Tabela 1), sendo excluídos os resumos dos artigos que não tinham obra alcançável e os trabalhos completos, empregando-se como palavras chave as sentenças, cuidados paliativos, doente terminal, assistência terminal, assistência paliativa e terminalidade da vida.

Dos 36 artigos científicos escolhidos, 1 trabalho foi publicado em 2000, 3 em 2001, 5 em 2002, 14 em 2003, 4 em 2004, 6 em 2005 e 3 em 2006, na atualidade houve um significativo aumento de publicações referentes a essa temática sobre os cuidados paliativos, mas neste trabalho foi focalizado publicações dos anos 2000 e 2006. Foram aplicados como fundamentos de 
incorporação os artigos que apresentavam as concepções dos cuidados paliativos, e pesquisas publicadas entre 2000 e 2006. Por conseguinte, foram obtidos cerca de 50 artigos relativos aos cuidados paliativos, sendo descartado os que não obedeciam ao processo definido. Por último, foram escolhidos 36 artigos, sendo estruturados em forma de fichas que se encontravam as informações dos artigos para compreender as concepções sobre cuidados paliativos.

Tabela 01: Artigos científicos analisados

\begin{tabular}{|c|c|c|}
\hline TITULOS DAS OBRAS PESQUISADAS & ANO DE PUBLICAÇÃO & SITES \\
\hline 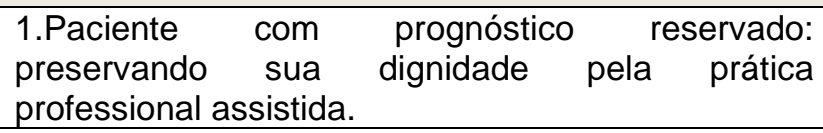 & 2000 & LILACS \\
\hline $\begin{array}{l}\text { 2. Assistência ao paciente terminal: alternativas } \\
\text { para o doente com AIDS }\end{array}$ & 2001 & LILACS \\
\hline $\begin{array}{l}\text { 3.Paciente terminal: ele deve ou não deve saber a } \\
\text { verdade? }\end{array}$ & 2001 & LILACS \\
\hline 4.El cuidado paliativo em casa al paciente terminal & 2001 & LILACS \\
\hline $\begin{array}{l}\text { 5. El morir y la muerte en la sociedad } \\
\text { contemporánea: problemas médicos y bioéticos. }\end{array}$ & 2002 & SciELO \\
\hline $\begin{array}{l}\text { 6. Morte e finitude em nossa sociedade: } \\
\text { implicações no ensino dos cuidados paliativos. }\end{array}$ & 2002 & LILACS \\
\hline 7. Cuidados paliativos oncológicos: controle da dor & 2002 & LILACS \\
\hline $\begin{array}{l}\text { 8. Intervenção psicoterapêutica na área de cuidados } \\
\text { paliativos para ressignificar a dor simbólica da } \\
\text { morte de pacientes terminais através do } \\
\text { relaxamento mental, imagens mentais e } \\
\text { espiritualidade. }\end{array}$ & 2002 & LILACS \\
\hline $\begin{array}{l}\text { 9. Cuidados paliativos oncológicos, controle de } \\
\text { sintomas: condutas do INCA }\end{array}$ & 2002 & LILACS \\
\hline 10. Spirituality and meaning in palliative care. & 2003 & LILACS \\
\hline $\begin{array}{l}\text { 11. Cuidados paliativos pediátricos: a essência do } \\
\text { cuidar da criança/adolescente/familiares nas } \\
\text { situações limites }\end{array}$ & 2003 & LILACS \\
\hline $\begin{array}{l}\text { 12. Comunicação nos programas de cuidados } \\
\text { paliativos: uma abordagem multidisciplinar. }\end{array}$ & 2003 & LILACS \\
\hline $\begin{array}{l}\text { 13. Um poeta fala nos últimos dias do ser humano: } \\
\text { introdução aos cuidados paliativos. }\end{array}$ & 2003 & LILACS \\
\hline 14. Os cuidados paliativos no Brasil & 2003 & LILACS \\
\hline $\begin{array}{l}\text { 15. Dor oncológica: bases para a avaliação e } \\
\text { tratamento. }\end{array}$ & 2003 & LILACS \\
\hline 16. A necessidade de cuidados paliativos. & 2003 & LILACS \\
\hline $\begin{array}{l}\text { 17. Comunicação com paciente fora de } \\
\text { possibilidades terapêuticas: reflexões. }\end{array}$ & 2003 & LILACS \\
\hline $\begin{array}{l}\text { 18. Análise dos registros de enfermagem sobredor } \\
\text { e analgesia em doentes hospitalizados. }\end{array}$ & 2003 & SciELO \\
\hline $\begin{array}{l}\text { 19. Cuidados paliativos: uma perspectiva de } \\
\text { assistência integral à pessoa com neoplasia. }\end{array}$ & 2003 & SciELO \\
\hline $\begin{array}{l}\text { 20. Assistência humanizada de enfermagem a } \\
\text { cliente em cuidados paliativos oncológicos: uma }\end{array}$ & 2003 & LILACS \\
\hline
\end{tabular}




\begin{tabular}{|l|c|l|}
\hline experiência com base em Jean Watson & 2003 & LILACS \\
\hline $\begin{array}{l}\text { 21. A comunicação do enfermeiro com cliente em } \\
\text { uma unidade de cuidados paliativos oncológicos no } \\
\text { serviço noturno }\end{array}$ & 2003 & LILACS \\
\hline $\begin{array}{l}\text { 22. Cuidados paliativos: uma necessidade urgente } \\
\text { na área de saúde }\end{array}$ & 2003 & LILACS \\
\hline 23. Enfermagem em cuidados paliativos & 2004 & SciELO \\
\hline 24. A morte no contexto dos serviços de saúde. & 2004 & SciELO \\
\hline $\begin{array}{l}\text { 25. A moralidade dos cuidados paliativos. } \\
\text { 26. Uma perspectiva histórica do sofrimento } \\
\text { humano: considerações Éticas no âmbito da saúde. }\end{array}$ & 2004 & SciELO \\
\hline $\begin{array}{l}\text { 27.Participação nas atividades de cuidados } \\
\text { paliativos a pacientes oncológicos }\end{array}$ & 2004 & LILACS \\
\hline $\begin{array}{l}\text { 28. Uma análise do conceito de cuidados paliativos } \\
\text { no Brasil }\end{array}$ & 2005 & LILACS \\
\hline $\begin{array}{l}\text { 29. Pacientes portadores de feridas neoplásicas em } \\
\text { serviços de cuidados paliativos: contribuições para } \\
\text { a elaboração de protocolos de intervenção de } \\
\text { enfermagem. }\end{array}$ & 2005 & SciELO \\
\hline $\begin{array}{l}\text { 30. O papel da fisioterapia nos cuidados paliativos } \\
\text { a pacientes com câncer. }\end{array}$ & 2005 & LILACS \\
\hline $\begin{array}{l}\text { 31. A Ética no cuidado durante o processo de } \\
\text { morrer: relato de experiência. }\end{array}$ & 2005 & LILACS \\
\hline $\begin{array}{l}\text { 32. Cuidados paliativos: aspectos bioéticos e } \\
\text { assistência de enfermagem ao paciente com dor }\end{array}$ & 2005 & SciELO \\
\hline $\begin{array}{l}\text { 33. Cuidados com o fim da vida: como abordar este } \\
\text { difícil tema? }\end{array}$ & 2005 & SciELO \\
\hline $\begin{array}{l}\text { 34. A finitude humana e a saúde publica } \\
\text { 35. Medicina paliativa: viejos conceptos: nuevos } \\
\text { escenarios }\end{array}$ & 2006 & SciELO \\
\hline $\begin{array}{l}\text { 36. Suporte ao paciente em cuidados paliativos em } \\
\text { pediatria }\end{array}$ & 2006 \\
\hline
\end{tabular}

Organização: OLIVEIRA, D. C., 2017

Posteriormente foram realizados trabalhos de campo na Associação Presente para observação in loco, entrevistas com a Dra. Priscila Bernardina Miranda (Presidente da Associação), usuários do Hóspice ${ }^{4}$, e a equipe multiprofissional, além de registros fotográficos.

\section{RESULTADOS E DISCURSÕES}

Com relação aos perfis dos periódicos no qual foram publicados os artigos, ocorreu a hegemonia com a apresentação de obras pertinentes aos atuais ramos da saúde numa concepção multidisciplinar, não havendo campo específico. Por conseguinte, as perspectivas sobre cuidados paliativos constam nos 36 artigos foram as seguintes: qualidade de vida, a apresentação humanística e reconhecimento da vida, o falecer como processo natural, o controle e atenuação da dor e dos demais sinais, as questões éticas, a abordagem multidisciplinar, a comunicação, a prioridade do cuidado e atenção

\footnotetext{
4 Lugar onde ocorre a realização da atividade dos Cuidados Paliativos com doentes em fase terminal busca conservar um ambiente familiar, com sociabilização dos pacientes, horário de visitas, autorização para o comparecimento de pessoas e animais.
} 
sobre a cura, a espiritualidade e o apoio no luto. Foi notável, nos trabalhos, que essas concepções estão intimamente interligadas e integradas.

Sobre a definição de cuidado paliativo, os artigos estudados ressaltam que a necessidade de cuidado paliativo não só acontece na etapa de terminalidade da vida, mas em todas as etapas da vida e, durante o desenvolvimento das enfermidades crônicas-degenerativas. Dessa maneira, muitas das concepções dos cuidados paliativos são utilizadas também, em fases iniciais da enfermidade, em junção com as terapias essenciais ao processo patológico, por compreender que a enfermidade, desde o seu começo, ocasiona impactos em variados aspectos no doente terminal. Deste modo, alguns princípios sobre cuidados paliativos obtidos foram:

A Qualidade de vida: No momento em que não há mais a probabilidade de cura, o centro do cuidado e da atenção ao doente terminal é a procura pela qualidade de vida na fase da finitude, que deve ser atingido por intermédio da proteção, conforto, atenuação e do monitoramento dos sintomas, tendo auxílio espiritual, psicossocial e ajuda no momento de enlutamento, ou seja, é fornecer qualidade de vida para o enfermo e família, na terminalidade da vida. A perspectiva de qualidade de vida, como algo intensamente essencial para paciente e seus familiares, está exposto em todas as obras estudadas, sendo o cuidado paliativo declarado como uma aproximação que aumenta a qualidade de vida. Segundo Pessini e Bertachini (2005, p. 496):

A questão da qualidade de vida tem atraído grande interesse de pesquisa nos últimos anos, uma vez que é importante reconhecer que tal fato não é simplesmente uma medida de conforto físico ou de capacidade funcional. Trata-se, antes, de algo que somente pode ser definido pela pessoa doente, e que pode ser alterado significativamente ao longo do tempo.

A apresentação humanista e reconhecimento da vida: A perspectiva de natureza especificamente humanista e filosófica como piedade, simplicidade, integridade e apreciação da vida manifestaram nas obras pesquisadas, dando respaldo à filosofia de cuidados paliativos como uma atenção afetiva e eficaz, com a utilização de critérios e ações que atendam e considerem o paciente como sujeito social, que possui uma carga de princípios pessoais, éticos, morais e religiosos. A evidenciação da importância da vida traz o princípio de que cada um tem uma proposito e exerce uma função relevante na sociedade, significando o ato de viver como uma "dádiva de Deus" e toda sua proporção. Assim, afirma Menezes (2004, p.10), "[...] humanização do morrer". Conforme Pessini e Bertachini (2005, p. 496), "um nível elevado de cuidado físico é certamente de vital importância, mas não suficiente em si mesmo. A pessoa humana não deve ser reduzida a uma mera entidade biológica".

O falecer como processo natural: Considera-se que o falecimento é a nossa realidade inevitável, e mesmo com o desenvolvimento do conhecimento cientifico que se dedica prorrogar a vida. A morte sendo considerada como um fenômeno inato e normal, em que todo o seu sentido deve ser debatido e desmitificado com o dente terminal, seus familiares e o profissional, estabelecendo o fenômeno de morrer menos angustiante, compreendendo em um ambiente de tranquilidade. Contudo, temos que entender que somos seres que fazemos parte de um sistema no qual estamos sujeitos às etapas que são: o nascer, o crescer, o reproduzir e o morrer. Conforme Pessini e Bertachini (2005), o que todos nós temos em comum é que em algum dia vamos falecer, e os cuidados paliativos possibilita uma finitude da vida de forma digna.

O controle e atenuação da dor e dos demais sinais: A percepção de um apropriado monitoramento e administração no abrandamento da dor e das demais manifestações da doença como objetivo principal dos cuidados paliativos manifestou em todos os artigos estudados. A dor aparece em pacientes que sofrem uma sucessão de incômodos de natureza física, psíquica, social e espiritual, por exemplo, as lesões cutâneas, odores desagradáveis, anorexia, insônia, fadiga, luto prévio, complicações econômicas, depressão, dentre outros. Pode se considerar que o suplício que a dor causa ao doente terminal traz consequências em todas as esferas tanto física, mental, social e espiritual e afeta também os familiares e os profissionais de saúde.

Em vista disso, o controle e atenuação da dor e dos demais sinais da doença é um direito do paciente e um dever dos profissionais de saúde, que devem elaborar métodos para amenizar o suplício ocasionado pela enfermidade. De acordo com Silva e Pimenta (2003, p. 110), "[...] os estudos evidenciam que a dor, quando não aliviada, limita o indivíduo nas atividades de vida diária, altera o 
apetite, o padrão de sono, a deambulação, a movimentação, o humor, o lazer, as atividades profissionais, sociais e familiares".

As questões éticas: Nos artigos estudados manifestaram-se diversas considerações de especificidade ética, que está presente nos cuidados paliativos, como por exemplo, algumas das concepções são destacadas na filosofia paliativista, através deste existem cinco princípios éticos relevantes que constitui a medicina paliativa, são conhecidos de princípios da veracidade (expor sempre a realidade ao doente e familiares), do equilíbrio terapêutico (somente aplicar critérios terapêuticos úteis), do duplo efeito (os resultados favoráveis devem ser superiores aos desfavoráveis), da prevenção (prognosticar dificuldades e orientar os familiares), e do não abandono (ser compassivo sempre, guiando o doente e a família). Temas como integridade no modo de falecer, liberdade para estabelecer sobre a vida, não redução da vida ou prorrogar de forma artificial, favorecer a caridade, a não perversidade e a justiça, também estão presentes nas obras pesquisadas.

Refletindo sobre estes assuntos, pode-se considerar que os cuidados paliativos procuram desenvolver a humanização na terminalidade da vida, por intermédio de ações que possibilita o morrer com integridade, orientado pelas concepções éticas de atenção a vida humana. Como Pessini e Bertachini (2005, p.498):

As questões éticas envolvidas em cuidados paliativos baseiam-se no reconhecimento do fato de que o paciente incurável ou em fase terminal não é um resíduo biológico por quem nada mais pode ser feito, um ser necessitado de anestesia, cuja vida não deve ser prolongada desnecessariamente. Estamos sempre diante de uma pessoa e, como tal, capaz - até o momento final — de relacionamento, de tornar a vida uma experiência de crescimento e de plenitude.

A abordagem multidisciplinar: A noção de um comportamento multidisciplinar para com o paciente e seus familiares apresenta um fato fundamental na filosofia paliativista, uma vez que são cuidados orientados para as manifestações físicas, sociais, psicológicas e espirituais, precisando de variados profissionais para tratar e zelar. Por conseguinte, o método multidisciplinar é primordial, visto que ameniza as dificuldades do doente e de seus familiares, por envolver inúmeros aspectos deve ser observado e analisado sob várias óticas procurando estabelecer um tratamento homogêneo e que tenha a finalidade que busca a terminalidade da vida de forma digna para o doente e sua família. Conforme a Academia Nacional de Cuidados Paliativos - ANCP (2012, p. 350), "[...] a atuação multiprofissional é essencial para que o paciente tenha qualidade de vida e uma sobrevida digna. Respeito, ética, sensibilidade e sinceridade devem sempre nortear a equipe durante o tratamento".

A comunicação: As obras pesquisadas relatam a magnitude de uma comunicação tanto verbal quanto não verbal, sincera entre o doente terminal, familiares e grupo profissional. Nesta perspectiva, a comunicação é essencial no relacionamento terapêutico que ocorre entre a equipe de especialistas e o enfermo e a família, visando estabelecer um elo de auxilio e cooperação eficaz, numa atmosfera apropriada, onde doente e seus familiares sejam capaz de manifestar e confessar temores, preocupação e princípios. É de extrema importância que o especialista tenha uma conduta honesta e franca, promovendo notícias e informações objetivas e autênticas, para que a convivência profissional-paciente ocorra com segurança. Segundo Moritz (2008, p. 424) comunicação:

[...] é a transmissão de informação continua de uma pessoa para outra, sendo então compartilhada por ambos. Para que haja uma comunicação, é necessário que o destinatário da informação receba e a compreenda. A informação simplesmente transmitida, mas não recebida, não foi comunicada; para tal, é necessário alinhar o transmissor e o receptor entre os participantes do processo. Na UTI, a comunicação é um processo que envolve a percepção do ambiente e do clima de trabalho, incluindo a comunicação nãoverbal da equipe multiprofissional, até a interação médico/paciente e família. Estão envolvidos no processo de comunicação na UTI os pacientes, seus familiares ou qualquer pessoa com proximidade afetiva, os médicos, enfermeiros, psicólogos, religiosos e os demais membros da equipe multiprofissional. 
A prioridade do cuidado e da atenção sobre a cura: Na filosofia da medicina paliativa, os cuidados paliativos são compreendidos como atenção pensada para o doente que não tem oportunidades terapêuticas, cuja não há mais possibilidade de cura, e que zelar torna-se uma prioridade. Por isso, o objetivo primordial da medicina paliativa é a atenção e a dedicação. Refletindo essa perspectiva pode inferir que a atenção e o cuidado devem ser separados da cura. As equipes de especialistas que acompanham o paciente em alguns casos preferem dar atenção à cura e quando a mesma não é atingida se considerada incapaz e deixa o cuidado em segundo plano. Sendo o cuidado de extrema essencial não só em momentos de finitude da vida, mas sim em todo viver. Com a atenção fica evidente $o$ respeito e a dignidade humana para com o paciente. De acordo com Oliveira e Silva (2010), nesse momento, de assistência paliativa os profissionais devem possibilitar ao máximo autonomia para o paciente.

A espiritualidade e o apoio no luto: Tanto o paciente que está em estado terminal quanto seus familiares, confrontam conteúdos de origem existencial, nos quais seus valores e princípios religiosos desempenham uma influente atuação no experienciar o processo de morte. Por este motivo manifesta a necessidade de atenuar o suplício de natureza espiritual, ao longo da doença e também no luto, por intermédio da ajuda da equipe de profissionais.

Contudo o luto é constituído por um processo em retorno a ruptura da ligação com determinada pessoa, é a percepção da ausência, um fenômeno extremamente doloroso. Por este aspecto, o luto apresenta grande relevância na filosofia de cuidados paliativos e foco de sua atividade. Conforme Pessini e Bertachini (2005, p. 496), "[...] em cuidados paliativos a família é uma unidade de cuidados; para tanto, as questões e dificuldades dos membros da família devem ser identificadas e trabalhadas. O cuidado com o luto se inicia bem antes do momento da morte do doente". Já para Caponero (2008, p. 12):

O luto é o processo de elaboração da perda de uma pessoa que sentimos próxima. As fases mais comuns nesse processo são o choque e a negação imediatos, as reações voláteis, a desorganização e desespero e, por fim, a reorganização. Como o diagnóstico, o luto também não começa de forma abrupta, com a morte. O processo de luto se inicia com a imaginação, ou constatação, de que a morte é uma potencialidade real. Nessas circunstâncias, não só as pessoas próximas desenvolvem reações de enlutamento frente ao doente, mas este também desenvolve uma reação de "luto invertido" composta basicamente pela elaboração das suas próprias perdas, e pela elaboração das mudanças no vínculo com o outro.

Através das observações in loco e questionários aplicados aos usuários dos serviços prestados pela Associação Presente. Percebeu-se que os pacientes utilizam infraestrutura e atendimento de boa qualidade, e ainda que a Associação Presente tenha um pequeno número de pacientes devido aos poucos meses de funcionamento direcionado especificamente para os cuidados paliativos que teve início em outubro de 2016, foi perceptível a melhoria dos pacientes.

Quando questionados sobre as atividades cotidianas realizadas e a autonomia foram unânimes em afirmar que após ter sido diagnosticado o câncer não puderam realizar suas práticas diárias básicas, e ainda afirmaram que se sentem incapazes, o paciente 1 chegou a utilizar o termo "[...] me sinto um inútil, por que sempre fui um homi trabalhador desde de molequim e hoje não consigo nem vestir uma roupa sozinho..."(SIC).

No momento em que foi perguntado a respeito da saúde houve algumas particularidades como na categoria "dor" todos falaram que sentem muitas dores e que atualmente estão tomando medicamentos; no quesito náuseas/enjoos ou vômitos o paciente 1 falou que sente mas, com pouca intensidade, os pacientes 2 e 3 disseram que não possuem esses sintomas; no parâmetro "falta de apetite e cansaço e/ou fraqueza" todos afirmaram que não tem sentido; já a categoria "falta de ar" nenhum possui; e a questão sobre "diarreia ou intestino preso" apenas o paciente 2 tem esta manifestação; e o último ponto questionado sobre a saúde foi " as alterações no sono" todos responderam que não observaram nenhuma mudança no sono. Segundo a Academia Nacional de Cuidados Paliativos - ANCP (2009, p.87), "[...] uma das importantes funções do sistema nervoso é fornecer informações sobre lesões corporais em potencial que são expressas pela dor. A percepção corporal da dor é denominada nocicepção". 
Na sexta pergunta realizada aos pacientes "Você tem autonomia para decidir seu final de vida?", nenhum quis responder, foi notado que eles possuem uma esperança de que no final vão conseguir atingir o milagre da cura, por meio de suas crenças. Quanto a "escolha do tratamento" o paciente 1 não quis responder e os paciente 2 e 3 afirmaram que o tratamento do jeito que está sendo encaminhado está muito bom e que não possuem a capacidade de escolher o esquema de tratamento. Já na questão sobre o "apoio" de parentes e/ou amigos e a equipe de assistência foram unanimes em dizer que recebem o amparo de todas as pessoas mais próximas e obtêm cuidados e atenção da equipe multiprofissional da Associação Presente.

A indagação sobre a "comunicação com o médico" foi observada que os pacientes não gostam muito de falar sobre possíveis problemas financeiros e profissionais e somente o paciente 1 falou que dialoga sobre relações sociais em geral e familiares. Ao serem questionados cerca dos "efeitos psicológicos" todos falaram que se sentem calmos e no controle da situação uma vez que isso ajuda a ter uma maior ciência da real situação em que se encontram; já no quesito "ser otimista" apenas o paciente 3 relatou que atualmente se encontra muito otimista com a sua situação, mas se define como uma pessoa realista; e na categoria "tristeza" o paciente 3 afirmou que está muito triste com o momento vivenciado; quando foi exposta a pergunta sobre "medo de morrer" o paciente 1 preferiu o silencio e os outros pacientes responderam que não tem medo da morte.

O momento final foi destinado para que falassem o que quiser. Neste período, eles dialogaram principalmente sobre o deslocamento constante deles da cidade que residem para a cidade de Montes Claros/MG, cujo percurso é muito longo e cansativo e foi exposto o receio de deixar a família em especial os filhos no falecimento. Conforme ANCP (2009, p.322), "[...] a partir do diagnóstico de uma doença potencialmente mortal seguido pela evolução da enfermidade, paciente e família deparam-se com rupturas, limitações e privações".

Em entrevista com a equipe multiprofissional, a médica oncologista e presidente da Associação Presente, informou que há 12 anos atua na instituição filantrópica. E quando questionada sobre "a sua percepção sobre os cuidados paliativos" ela respondeu que é "Uma grande área da medicina capaz de levar acolhimento, conforto e dignidade a quem tem a vida ameaçada por uma doença incurável como é o câncer". Marciel et al (2006, p. 12) apontam que:

Cuidados paliativos são: cuidados ativos e integrais prestados a pacientes com doença progressiva e irreversível, com poucas chances de resposta a tratamento curativo, sendo fundamental o controle da dor e de outros sintomas através da prevenção do alivio do sofrimento físico, psicológico, social e espiritual. Cuidados prestados por equipes multiprofissionais, em ambientes hospitalar ou domiciliar, segundo níveis de diferenciação que devem incluir, ainda, o apoio à família e atenção ao luto.

Para a Psicóloga "os cuidados paliativos no Brasil são muito recentes, que no Norte de Minas temos somente a Associação Presente, cujo atendimento é muito diferenciado dos atendimentos convencionais em tratamento de câncer, é fundamental, pois, a atenção paliativa valoriza a vida e que o objetivo é oferecer o cuidado com qualidade e respeito ao paciente". Já para a Nutricionista é "é o apoio ao paciente no aspecto físico, social, emocional e espiritual, não somente no processo do adoecimento, mas também no luto com os familiares".

Posteriormente a presidente da Associação Presente explicou com é feito a seleção dos pacientes, ela informa que "Os pacientes procuram por eles próprios, uma vez que nenhum dos dois serviços de referência do tratamento do câncer disponibilizam ambulatório de Cuidados Paliativos". Foi comentado também que a instituição presta atendimentos domiciliares sempre que necessário. $E$ os principais procedimentos que são empregados no atendimento aos pacientes são o alivio da dor, fadiga, dispneia e caquexia.

Ainda em entrevista a mesma comentou que "além da dor, no dia a dia, outro problema enfrentado pelos enfermos é a desinformação, pois poucos profissionais se sentem confortáveis para partilhar a verdade do prognóstico com seus pacientes". Quando feita a indagação sobre como é a aceitação dos pacientes frente ao processo de terminalidade da vida, ela ressalta que "quando comunicado de maneira acolhedora, transparente e amorosa, os pacientes experimentam dor, lágrimas, mas são capazes de repensarem algumas escolhas e organizarem suas vidas, valorizando mais o tempo e os laços de amor". 
Foi questionado sobre a infraestrutura na Associação Presente para os cuidados paliativos aos pacientes se é adequada ou se ainda necessita de melhorias, ela complementa "para o ambulatório sim. Mas, sonhamos em construir o primeiro hospice do interior de Minas Gerais". Foi levantado a discursão acerca das principais dificuldades enfrentadas pela equipe no cuidado a esses pacientes na Associação Presente, a médica respondeu que "a desinformação e o medo (do paciente sobre a morte e da família sobre o luto)". E já os principais obstáculos para implementar a instituição e como conseguiram contorna-los, ela afirmou que foi "o financeiro (ainda estamos enfrentando...por isso não começamos a obra ainda) e o outro cultural (no Brasil, não conversamos sobre a morte)".

Ao ser questionado se ela tem conhecimento de outros locais no Norte de Minas onde funciona um hospice em Cuidados Paliativo, ela afirmou que "não existe, seremos o primeiro". No final foi proposto na entrevista para falar sobre sua trajetória em cuidados paliativos (razões que motivaram esse cuidado), ela pontua que, "no dia da defesa do mestrado (08/12/2010), recusei o doutorado ao componente da banca por absoluto cansaço físico-mental. Ao chegar em casa havia um embrulho grande que era um presente de um paciente querido. Quando abri haviam 7 livros cujos temas eram sobre Cuidados Paliativos. Apaixonei-me por essa medicina humana, verdadeira que permite vir a tona todas as emoções e oportunidades que a percepção da finitude nos traz. Devorei os livros em poucos dias e 2 meses depois (2011) iniciei um curso de pós-graduação da Casa do Cuidar em São Paulo. Em 2012 fiz outro curso de 6 meses, de controle de sintomas e em 2014 fui para Harvard Medical School fazer nova pós-graduação em Cuidados Paliativos. Em 2015 abri o primeiro ambulatório de Cuidados Paliativos da cidade."

Ainda conclui que "Amo os Cuidados Paliativos em especial porque ele me permite ser quem sou e cuido de seres humanos que não estão mais preocupados com imagem. Essas pessoas têm sede de vida, pois o tempo lhes urge e enquanto a morte não chega aprendem que é maravilhoso viver e celebrar a vida!".

Com base nos princípios expostos e nas atividades in loco na Associação Presente, verifica-se que no caso dessa Associação, os cuidados paliativos são direcionados exclusivamente para a oncologia, que se dedica aos enfermos de todas as faixas etárias, a partir das necessidades particulares de cada paciente. As atividades de cuidados paliativos têm como objetivo principal adquirir a mais satisfatória qualidade de vida para cada doente na finitude da vida e seus familiares que implicam: no cuidado e auxilio no monitoramento e gestão dos sinais e a utilização de um comportamento totalizante tendo como foco as experiências vivenciadas pelo paciente e a sua realidade atual. Refere-se a uma atenção e auxilio ao paciente na fase final da vida e familiares necessitando de uma comunicação sincera e humanitária por parte da equipe multiprofissional.

\section{CONSIDERAÇÕES FINAIS}

À medida que o movimento filosófico comprometido com a atenuação do suplício do doente com enfermidades crescentes e aterrorizantes da conservação existencial, os Cuidados Paliativos se desenvolvem com paradigmas científicos que apresenta os objetos de estudo que são: o processo saúde-doença, o cuidado e a atenção considerando a sua história e dignidade do paciente. $\mathrm{O}$ auxilio paliativo valoriza a arte do zelar.

A partir dos trabalhos científicos estudados a as entrevistas in loco na Associação Presente de Apoio a Pacientes com Câncer - Padre Tiãozinho, no Norte de Minas Gerais, foi possível constatar a relevância dos cuidados paliativos na assistência aos doentes carentes sem probabilidades terapêuticas curativas, no qual o processo de zelar é extremamente importante. Entretanto, mesmo com o auxílio, o paciente pode vivenciar temor, o isolamento ou o declínio da autoestima. A equipe multiprofissional da instituição filantrópica, nota que, para além do controle dos sinais físicos, a ideologia dos cuidados paliativos se empenha com a qualidade e valorização da vida dos pacientes.

A atuação do grupo de especialistas em Cuidados Paliativos é fundamental, tratando sempre com ética e dignidade os pacientes. A comunicação verbal e a não-verbal comprovam componentes substanciais à demarcação e a legitimação da autonomia dos pacientes sem expectativas curativas, e intensificam encaminhamento do processo terapêutico face às necessidades individuais e humanas. Neste contexto, a Associação Presente tem atuado no Norte de Minas Gerais com qualidade, para sanar as necessidades dos pacientes carentes de modo humanizado, possibilitando atividades que assegurem uma sobrevida íntegra e atenuação apropriada dos sinais físicos, psicológicos e 
espirituais, em concordância com a ideologia paliativista, entendendo que o paciente e seus familiares necessitam de amparo, cuidado e atenção.

\section{REFERÊNCIAS}

ÁLVAREZ, E.T. El cuidado paliativo em casa al paciente terminal. IN: MedUNAB. P. 82-92,2001.

ABCP. Associação Brasileira de Cuidados Paliativos. Disponível em: < www.cuidadospaliativos.com.br> acesso em: 20/12/16.

ANCP. Manual de Cuidados Paliativos. Rio de Janeiro: Diagraphic, 2009.

ANCP. Manual de Cuidados Paliativos ANCP. 2 eds. São Paulo: ANCP,2012.

ANCP. Academia Nacional de Cuidados Paliativos. Disponível em: < www.paliativo.org.br $>$ acesso em: 20/12/16.

ARAÚJO, Noemi. Assistência humanizada de enfermagem a cliente em cuidados paliativos oncológicos: uma experiência com base em Jean Watson. Rio de Janeiro:s,n. 2003.

BRASIL. Ministério da Saúde. Instituto Nacional de Câncer. Cuidados paliativos oncológicos: controle da dor. Rio de Janeiro: INCA, 2001.

BRASIL. Ministério da Saúde. Instituto Nacional de Câncer. Cuidados paliativos oncológicos, controle de sintomas: condutas do INCA. IN: Rev bras control .Abr-jun,p.191-211,2002.

BREITBART, W. Spirituality and meaning in palliative care.IN: Mundo Saúde (1995); 27(1), p.33-44, 2003.

CÂMARA, Ana Maria Chagas Sette, et al. Percepção do Processo Saúde-doença: Significados e Valores da Educação em Saúde. IN: Revista Brasileira de Educação Médica. 36 (1, Supl. 1), P.4050; 2012. https://doi.org/10.1590/S0100-55022012000200006

CAPELAS, Manuel Luís Vila - Cuidados Paliativos: uma proposta para Portugal. IN: Cadernos de Saúde. Lisboa, V. 2,N. P. 51-57, 2009.

CAPONERO, Ricardo. Natureza da angústia nos pacientes com Neoplasia. IN: Revista brasileira de cuidados paliativos. São Paulo, ano 01, ํํ 01, v. 01. P. 09-13, 2008.

CARVALHO, M.V.B, PERINA, E.M. Cuidados paliativos pediátricos: a essência do cuidar da criança/adolescente/familiares nas situações limites. IN:Mundo Saúde (1995).; 27(1): p.93-7, 2003.

CARVALHO, Mara Villas Boas de. Paciente com prognóstico reservado: preservando sua dignidade pela prática professional assistida.IN: Rev. ciênc. Méd.Campinas;p.86-90, 2000.

COSTA S.M.M, et al. Cuidados paliativos em neonatologia: implementação de um programa multiprofissional. In:Mundo Saúde (1995).; 27(1): p.171-6, 2003.

ELIAS, A.C.A.; GIGLIO, J.S. Intervenção psicoterapêutica na área de cuidados paliativos para ressignificar a dor simbólica da morte de pacientes terminais através do relaxamento mental, imagens mentais e espiritualidade.IN: Revista psiquiatr.clinc. São Paulo:p. 116-129,2002.

FIRMINO, F. Pacientes portadores de feridas neoplásicas em serviços de cuidados paliativos: contribuições para a elaboração de protocolos de intervenção de enfermagem. IN:Rev Bras Cancerol.; 51(4); p.347-59, 2005.

GOMES, A.P.R, ALMEIDA, H.O. A morte no contexto dos serviços de saúde. IN:Rev Saude, Dist Fed.; 15(1/2): p.19-32, 2004.

GUERRA, Maria Aparecida Telles. Assistência ao paciente terminal: alternativas para o doente com AIDS. Sã Paulo: s.n,2001.

JAYME, Naibi Souza. Geografia do Câncer: Espacialização dos Casos de Neoplasia Ocupacional na Macrorregional de Saúde do Norte do Estado do Paraná, entre o período de 2001 a 2011. 122 f.

Trabalho de Conclusão de Curso (Bacharelado em Geografia) - Universidade Estadual de Londrina, Londrina, 2013. https://doi.org/10.12957/tamoios.2014.9767 
KAVALEC,F.L. Participação nas atividades de cuidados paliativos a pacientes oncológicos. Curitiba:s.n., 2004.

KOVÁCS,M.J. Bioética nas questões da vida e da morte. IN:Psicol USP.; 14(2): p.115-67, 2003. https://doi.org/10.1590/S0103-65642003000200008

KOVÁCS, M.J. Comunicação nos programas de cuidados paliativos: uma abordagem multidisciplinar. IN:Mundo Saúde (1995).; 27(1): p.71-80, 2003.

KURASHIMA,A.Y.;DE CAMARGO,B. Suporte ao paciente em cuidados paliativos em pediatria.IN: Manual de Condutas Diagnosticas e Terapêuticas em Oncológicas. São Paulo: Âmbito Editores, 3 ed, p.218-222, 2006.

LAGO,P.M.; LOPES, M.H.I. Cuidados com o fim da vida: como abordar este difícil tema?.IN: Sci. Med. P.47-51,2005.

LEPARGNEUR, H. Um poeta fala nos últimos dias do ser humano: introdução aos cuidados paliativos. IN:Mundo Saúde (1995).; 27(1): p.185-9, 2003.

MARCIEL, Maria Goretti Sales; et al. Critérios de qualidade para os cuidados paliativos no Brasil / documento elaborado pela Academia Nacional de Cuidados Paliativos. Rio de Janeiro: Diagraphic, 2006.

MARCUCCI, F.C.I. O papel da fisioterapia nos cuidados paliativos a pacientes com câncer. IN:Rev Bras Cancerol.; 51(1),p..67-77, 2005.

MELO, A.G.C. Os cuidados paliativos no Brasil. IN:Mundo Saúde (1995); 27(1): p.58-63,2003.

MENEZES. R. A. Em busca da boa morte: antropologia dos cuidados paliativos. Rio de Janeiro: Garamond, FIOCRUZ, 2004.

e HEILBORN, M. L. A inflexão de gênero na construção de uma nova especialidade médica. IN: Estudos Feministas.Florianópolis, 15(3), p. 563-580, set./dez., 2007. https://doi.org/10.1590/S0104-026X2007000300004

MORITZ, Rachel Duarte; et al. Terminalidade e cuidados paliativos na unidade de terapia intensiva. IN: Rev Bras Ter Intensiva.20 (4). São Paulo, p. 422-428; 2008. https://doi.org/10.1590/S0103$\underline{507 X 2008000400016}$

MS (MINISTÉRIO DA SAÚDE). Portaria GM/MS n. ${ }^{\circ} 19$ de 3 de Janeiro de 2002.

MS (MINISTÉRIO DA SAÚDE). Portaria GM/MS n. ${ }^{\circ} \mathbf{2 . 4 3 9}$ de 8 de dezembro de 2005.

MS (MINISTÉRIO DA SAÚDE). Portaria GM/MS n. ${ }^{\circ} \mathbf{3 . 1 5 0}$ de 12 de dezembro de 2006.

MCCOUGHLAN, M. A necessidade de cuidados paliativos.IN: Mundo Saúde (1995); 27(1): p. 6-14, 2003.

MUÑOZ,AL.; TAPIA,I.P. Medicina paliativa: viejos conceptos: nuevos escenarios. IN:Rev Hosp. Clin.Uni.Chile. p.158-164,2006.

OLIVEIRA, Aline Cristine de; SILVA, Maria Júlia Paes da. Autonomia em cuidados paliativos: conceitos e percepções de uma equipe de saúde. IN: Acta Paulista de Enfermagem, v.23, n.2, p.212-217, 2010. https://doi.org/10.1590/S0103-21002010000200010

OLIVEIRA,J.B.A.; MORAES,M.I,M. Paciente terminal deve ou não deve saber a verdade?.IN: J.bras med.;81(2),p.47-9,ago,2001.

ORGANIZAÇÃO MUNDIAL DA SAÚDE - Programas nacionais de controle do câncer: políticas e diretrizes gerenciais. Genebra: Organização Mundial de Saúde, 2002.

PESSINI, L.; CAPONERO,R.; MELO, A. G.C.Cuidados paliativos: uma necessidade urgente na área de saúde. IN:Mundo Saúde.p.3-5,jan-mar,2003.

PESSINI, Leo; BERTACHINI, Luciana. Novas perspectivas em cuidados paliativos: ética, geriatria, gerontologia, comunicação e espiritualidade.IN: O Mundo da Saúde.São Paulo, V. ano 29 v. 29 n. 4 out./dez.P. 491-509, 2005. 
PESSINI, L. Distanásia: até quando prolongar a vida? São Paulo: Centro Universitário São Camilo e Edições Loyola, 2001.

Cuidados Paliativos: alguns aspectos conceituais, biográficos e éticos. Rev. Prática Hospitalar, 7(41), 2005. Disponível em: http://www.praticahospitalar.com.br/pratica\%2041/pgs/materia\%2021-41.html. Acesso em 20/12/ 20016.

A filosofia dos cuidados paliativos: uma resposta diante da obstinação terapêutica. In: PESSINI, L.; BERTACHINI, L. (Orgs.) Humanização e Cuidados Paliativos. São Paulo; Centro Universitário São Camilo e Edições Loyola, p. 181-208, 2006.

PIMENTA, C.A.M. Dor oncológica: bases para a avaliação e tratamento. IN:Mundo Saúde (1995). ; 27(1): p.98-110, 2003.

PONTES,M. G. A comunicação do enfermeiro com cliente em uma unidade de cuidados paliativos oncológicos no serviço noturno. Rio de Janeiro: s.n.,2003.

REGO, S, Palácios M. A finitude humana e a saúde publica. IN: Cad Saúde Publica = Rep Public Health.; 22(8):p.175-560, 2006. https://doi.org/10.1590/S0102-311X2006000800025

RODRIGUES, I.G, ZAGO, M.M.F, CALIRI, M,H. Uma análise do conceito de cuidados paliativos no Brasil. IN:Mundo Saúde (1995).; 29(2): p.147-54, 2005.

RODRIGUÉZ, E. V. El morir y la muerte en la sociedad contemporánea: problemas médicos y bioéticos. IN:Revista Gerencia y Políticas de Salud, P.66-79, set. 2002.

RODRIGUES,I. G.; ZAGO,M.M. F. Enfermagem em cuidados paliativos. IN: Mundo Saúde.p. 8292,jan-mar,2003.

SALES, Catarina Aparecida and Alencastre, Márcia Bucchi. Cuidados paliativos: uma perspectiva de assistência integral à pessoa com neoplasia.IN: Rev. bras. enferm., vol.56, no.5, p.566-569,2003. https://doi.org/10.1590/S0034-71672003000500020

SAMPAIO,F.A. Cuidados paliativos: aspectos bioéticos e assistência de enfermagem ao paciente com dor. Rio de Janeiro: s.n.,2005.

SCHRAMM, F.R. Morte e finitude em nossa sociedade: implicações no ensino dos cuidados paliativos.IN: Rev Bras Cancerol; 48(1):p. 17-20,2002.

SILVA, Yara Boaventura da; PIMENTA, Cibele Andrucioli M. Análise dos registros de enfermagem sobredor e analgesia em doentes hospitalizados.IN. Rev Esc Enferm USP, São Paulo, 37(2): p.10918,2003. https://doi.org/10.1590/S0080-62342003000200013

SILVA, C.H.D. A moralidade dos cuidados paliativos.IN:Rev Bras Cancerol.; 50(4):p. 330-3, 2004.

SILVA,M.J.P. Comunicação com paciente fora de possibilidades terapêuticas: reflexões. IN:Mundo Saúde (1995). ; 27(1): p.64-70, 2003.

SOUZA, L.B, SOUZA, L.E.E.M, SOUZA, A.M.A. A Ética no cuidado durante o processo de morrer: relato de experiência. IN:Rev Bras Enferm.; 58(6): p.731-4, 2005. https://doi.org/10.1590/S0034$\underline{71672005000600020}$

TRINDADE, E.M.V. Uma perspectiva histórica do sofrimento humano: considerações Éticas no âmbito da saúde. IN:Rev Saúde, Dist Fed; 15(1/2):p. 9-18, 2004.

WHO. The World Health Report 2001: Mental Health: New Understanding, Genebra, Suíça: New Hope, 2001.

WHO. Cancer pain relief and Palliative Care: Report of a WHO Expert Committee, 1990.

WHO. National cancer control programmes: policies and managerial guidelines. 2rd ed. Geneve: OMS; 2002.

WHO. WHO definition of Palliative Care. Disponível em:

<http://www.who.int/cancer/palliative/definition/en> Acesso em: 20/12/ 2016. 